\title{
Downregulation of NIMA-related kinase-7 inhibits cell proliferation by inducing cell cycle arrest in human retinoblastoma cells
}

\author{
JIAN ZHANG $^{1}$, LI WANG ${ }^{2}$ and YONGKANG ZHANG ${ }^{3}$ \\ ${ }^{1}$ Department of Ophthalmology, Shaanxi Provincial People's Hospital, Xi'an, Shaanxi 710068; \\ ${ }^{2}$ Department of Ophthalmology, Ankang City Central Hospital, Ankang, Shaanxi 725000; ${ }^{3}$ Department of \\ Ophthalmology, Shaanxi Provincial Rehabilitation Hospital, Xi'an, Shaanxi 710065, P.R. China
}

Received August 22, 2016; Accepted May 5, 2017

DOI: 10.3892/etm.2017.5558

\begin{abstract}
NIMA-related kinase-7 (Nek7) is a centrosomal kinase involved in various types of cancer, including gallbladder cancer and hepatocellular carcinoma. However, the biological function and the potential underlying mechanism of Nek7 in retinoblastoma remain largely unknown. Therefore, the present study investigated the effects of Nek7 in retinoblastoma cells. The expression of Nek7 was initially determined and observed to be commonly upregulated in retinoblastoma cell lines (Y79, SO-RB50 and WERI-RB1) as compared with that in normal retinal pigment epithelium cells. Next, the endogenous expression of Nek7 was efficiently knocked down in Y79 and SO-RB50 cells using a lentivirus-mediated RNA interference approach, as confirmed by reverse transcription-quantitative polymerase chain reaction and western blot analysis. Loss-of-function assays, including MTT, colony formation and flow cytometry, indicated that knockdown of Nek7 significantly inhibited cell growth, impaired the colony formation ability and induced cell cycle arrest at G0/G1 phase. Furthermore, mechanistic studies demonstrated that silencing of Nek7 resulted in reduced cyclin-dependent kinase 2, cyclin D1 and cyclin E levels in vitro. In conclusion, the present study highlights the crucial role of Nek7 in promoting retinoblastoma cell proliferation, and Nek7-silencing may serve as a novel therapeutic target for retinoblastoma.
\end{abstract}

\section{Introduction}

Retinoblastoma is the most common intraocular malignancy that usually occurs in childhood, resulting from genetic

Correspondence to: Dr Yongkang Zhang, Department of Ophthalmology, Shaanxi Provincial Rehabilitation Hospital, 52, 2nd Electronic Road, Xi'an, Shaanxi 710065, P.R. China

E-mail: ykzhang_123@163.com

Key words: NIMA-related kinase-7, retinoblastoma, cell proliferation, cell cycle alterations and transformation of mature retinal cells (1), and is estimated to account for $3-4 \%$ of all pediatric cancer cases (2). Retinoblastoma is derived from the inner neuroblastic and neuroblastic layer cells of the retina (3), and often extends into the brain along the optic nerve to easily metastasize distally (4). Currently, multidisciplinary management strategies, including enucleation, radiation therapy and chemotherapy, are effective therapies for children with retinoblastoma $(5,6)$; however, these methods present resultant short- and long-term adverse effects. Therefore, investigating the biology and molecular mechanism underlying the initiation and progression of retinoblastoma would assist in the development of novel therapeutic targets for retinoblastoma treatment.

The mammalian NIMA-related kinase (Nek) family includes 11 structurally conserved proteins, namely Nek1-11, which were initially identified as human orthologs to the founder kinase, NIMA $(7,8)$. NIMA has been reported to serve an important role in the reorganization of the microtubule network observed during mitosis by enabling the entrance of $\mathrm{Cdc} 2 /$ cyclin B complex into the nucleus (9), thus suggesting that NIMA is correlated with mitotic progression and cell cycle regulation $(10,11)$. The Nek6/7 subgroup, consisting of a core kinase domain and a short N-terminal tail, includes the smallest Nek proteins that are widely expressed in tissue-specific complimentary patterns during embryonic development $(12,13)$. It has been recently demonstrated that depletion of Nek6 by siRNA induced cell cycle arrest and apoptosis (14). Notably, the kinase domain of Nek7 is $\sim 87 \%$ similar to that of Nek6, and Nek7 is required for proper spindle assembly and mitotic progression (15), which suggests it may serve an important role in regulating cell proliferation. In fact, Nek7 has been reported to be highly expressed and to promote tumor cell proliferation in vitro in gallbladder cancer (16) and hepatocellular carcinoma (17). Furthermore, a recent study by Kooi et al (18) has identified Nek7 as a novel retinoblastoma candidate driver gene with high expression, suggesting that Nek7 may exert important effects on the progression of retinoblastoma. However, the biological function of Nek7 and its potential underlying mechanism in retinoblastoma have not been investigated. 
In the present study, the expression of Nek7 in different retinoblastoma cell lines was initially determined and compared with that in normal cells. Subsequently, the potential role of Nek7 in retinoblastoma cell proliferation was investigated using a lentivirus-mediated specific short hairpin RNA (shRNA) targeting Nek7. The findings of the current study will contribute towards improving the understanding on the molecular mechanisms of retinoblastoma cell growth and development.

\section{Materials and methods}

Cell lines and culture. A human retinoblastoma cell line Y79 (cat. no. HTB-18 ${ }^{\mathrm{TM}}$ ) and a normal retinal pigment epithelium (RPE) cell line (cat. no. CRL-4000 ${ }^{\mathrm{TM}}$ ) were purchased from American Type Culture Collection (Manassas, VA, USA). The human retinoblastoma cell line SO-Rb50 (cat. no. TCHu213; Cell Bank of Chinese Academy of Science, Shanghai, China) was obtained from the Department of Pathology of the Zhongshan Ophthalmic Center, Sun Yat-sen University (Guangzhou, China). The human retinoblastoma cell line WERI-RB1 (cat. no. TCHu213) and 293T cells (cat. no. GNHu17) were purchased from the Cell Bank of Chinese Academy of Science. 293T cells were cultured in Dulbecco's modified Eagle's medium (Hyclone; GE Healthcare Life Sciences, Logan, UT, USA), supplemented with $10 \%$ fetal bovine serum (FBS; Gibco; Thermo Fisher Scientific, Inc., Waltham, MA, USA) with $100 \mu \mathrm{g} / \mathrm{ml}$ penicillin/streptomycin. Human retinoblastoma and RPE cells were cultured in RPMI 1640 (Hyclone; GE Healthcare Life Sciences) medium supplemented with $10 \%$ FBS. All cells were maintained at $37^{\circ} \mathrm{C}$ in a humidified incubator with $5 \% \mathrm{CO}_{2}$.

RNA isolation and reverse transcription-quantitative polymerase chain reaction ( $R T-q P C R)$. Total RNA was extracted from human retinoblastoma cells using TRIzol reagent (Invitrogen; Thermo Fisher Scientific, Inc.) according to manufacturer's protocols. All RNA was quantified by using the Nanodrop spectrophotometer ND-2000 (Thermo Fisher Scientific, Inc., Wilmington, DE, USA). Only those RNA samples with 260/280 ratios of 1.8-2.0 were used for further investigation. Then, cDNA was synthesized using M-MLV Reverse Transcriptase (cat. no. 28025013; Invitrogen; Thermo Fisher Scientific, Inc.) according to manufacturer's protocols. Next, a SYBR GreenER ${ }^{\mathrm{TM}}$ qPCR SuperMix Universal kit (cat. no. 11762-100; Invitrogen, Thermo Fisher Scientific, Inc.) was used to determine the mRNA level of Nek7 in human retinoblastoma and RPE cells using a CFX96 Touch ${ }^{\mathrm{TM}}$ Real-Time PCR detection system (Bio-Rad Laboratories, Inc., Hercules, CA, USA). Primers used for amplification were as follows: Nek7 (forward), 5'-CACCTGTTCCTCAGTTCCAAC-3'; Nek7 (reverse), 5'-CTCCATCCAAGAGACAGGCTG-3'; $\beta$-actin (forward), 5'-GTGGACATCCGCAAAGAC-3'; and $\beta$-actin (reverse), 5'-AAAGGGTGTAACGCAACTA-3'. The PCR protocol was as follows: Initial denaturation at $95^{\circ} \mathrm{C}$ for $60 \mathrm{sec}, 40$ cycles of denaturation at $95^{\circ} \mathrm{C}$ for $5 \mathrm{sec}$, annealing and extension at $60^{\circ} \mathrm{C}$ for $20 \mathrm{sec}$. The relative Nek7 expression levels were calculated using the $2^{-\Delta \Delta \mathrm{Cq}}$ method (19). $\beta$-actin was used as an internal control.
Western blot analysis. Cells were washed twice with ice-cold phosphate-buffered saline (PBS) and lysed with radioimmunoprecipitation assay buffer $(50 \mathrm{mM}$ Tris- $\mathrm{HCl}, \mathrm{pH} 7.4,150 \mathrm{mM}$ $\mathrm{NaCl}, 1 \% \mathrm{NP}-40$ and $0.1 \% \mathrm{SDS}$ ) containing $1 \mathrm{mM}$ phenylmethane sulfonyl fluoride, a serine/cysteine protease inhibitor (cat. no. ST506; Beyotime Institute of Biotechnology, Haimen, China). The supernatant was obtained for protein concentration determination by the BCA Protein Assay kit (cat no. 23235; Pierce; Thermo Fisher Scientific, Inc.). Samples of equal amount of protein were separated by 10-12\% SDS-PAGE, and the resulting bands were transferred to polyvinylidene difluoride microporous membranes (EMD Millipore, Billerica, MA, USA). The membrane were blocked in 5\% non-fat milk in Tris-buffered saline with Tween-20 (TBS-T) buffer for $1 \mathrm{~h}$ and then incubated overnight at $4^{\circ} \mathrm{C}$ with the following primary antibodies: Rabbit anti-Nek7 (cat. no. 3057; 1:1,000 dilution), cyclin D1 (cat. no. 2978; 1:1,000 dilution), cyclin-dependent kinase 2 (CDK2; cat. no. 2546; 1:1,000 dilution), cyclin E (cat. no. 20808; 1:1,000 dilution) and GAPDH (cat. no. 2118; 1:4,000 dilution) (all Cell Signaling Technology, Inc., Danvers, MA, USA). Subsequently, samples were washed three times with TBS-T buffer and incubated with goat anti-rabbit secondary antibodies conjugated with horseradish peroxidase (cat. no. 7074; 1:5,000 dilution; Cell Signaling Technologies, Inc.) for $1 \mathrm{~h}$ at $37^{\circ} \mathrm{C}$. Signal intensity was detected using an ECL detection system (Pierce; Thermo Fisher Scientific, Inc.). GAPDH was used as the reference control.

Construction of lentivirus vector and transduction of retinoblastoma cells. The stem-loop-stem oligos were synthesized by Sangon Biotech Co., Ltd. (Shanghai, China), including two Nek7 shRNAs (shNek7-1, 5'-GGAUGAGCA AUCACAAGGA-3'; and shNek7-2, 5'-GCUAAUUCCUGA AAGAACU-3') and a negative control shRNA (shNC), 5'-CGU CAGAGUAUACUAAUAU-3'. These oligos were annealed and ligated into the pLKO.1 vector (Addgene, Inc., Cambridge, MA, USA) according to the instructions provided by the manufacturer. Lentiviruses were generated by triple transfection of $80 \%$ confluent $293 \mathrm{~T}$ cells with $8 \mu \mathrm{g}$ shNek7-1/pLKO.1, shNek7-2/pLKO.1 or shNC/pLKO.1, along with packaging pHelper plasmids (pVSVG-I and pCMVAR8.92) using Lipofectamine 2000 (Invitrogen, Thermo Fisher Scientific, Inc.) according to the manufacturer's procedure. The supernatant was collected $48 \mathrm{~h}$ after transfection and lentiviral particles were harvested by ultracentrifugation (speed, $4,000 \times \mathrm{g}$ ) at $4^{\circ} \mathrm{C}$ for $10 \mathrm{~min}$ followed by filtration through a $45 \mu \mathrm{m}$ filter, and the titer of lentiviruses was measured as previously described (20).

For cell transfection, the human retinoblastoma cell lines Y79 and SO-RB50 were transfected using Lipofectamine 2000 with $5 \mu \mathrm{g}$ constructed lentiviruses containing shNek7-1, shNek7-2 or shNC, respectively at $37^{\circ} \mathrm{C}$ in a humidified incubator with $5 \% \mathrm{CO}_{2}$. After $96 \mathrm{~h}$ of transfection, cells were collected and the transfection efficiency was determined by examining the green fluorescence protein (GFP) expression (Addgene, Inc.) through a fluorescence microscope at x100 magnification, while the knockdown efficiency was determined by RT-qPCR and western blot analysis.

Cell viability assay. Cell growth curves were determined using the MTT method. Briefly, following transfection as described 
earlier, Y79 and SO-RB50 cells were reseeded in 96-well plates at a density of 3,000 cells/well. The medium containing $0.5 \mathrm{mg} / \mathrm{ml}$ MTT was added into each well and then incubated at $37^{\circ} \mathrm{C}$ in a humidified $5 \% \mathrm{CO}_{2}$ atmosphere for $4 \mathrm{~h}$. This was followed by the addition of $150 \mu \mathrm{l}$ solubilization solution $(0.01 \mathrm{M} \mathrm{HCl}, 10 \%$ SDS and 5\% isopropanol) to each well and incubation of cells for a further $10 \mathrm{~min}$ at $37^{\circ} \mathrm{C}$ with gentle shaking. The optical density of the plates was measured using an Epoch Microplate Spectrophotometer (BioTek Instruments, Inc., Winooski, VT, USA) at an absorbance of $595 \mathrm{~nm}$.

Colony formation assay. Y79 and SO-RB50 cells were reseeded in 6-well plates at a density of 500 cells/well after 4 days of lentivirus transfection. Media were replaced every 3 days. After 8 days of culture, the cells were washed with PBS and fixed with PBS containing 4\% paraformaldehyde for $30 \mathrm{~min}$ at room temperature. Next, fixed cells were stained with $1 \%$ crystal violet (cat no. C0121; Beyotime Institute of Biotechnology) for 10 min, gently washed with $\mathrm{ddH}_{2} \mathrm{O}$ and air dried. Images of the 6 -well plates were captured with a camera, and visible colonies with $>50$ cells were counted under a microscope. Image analysis was conducted using Image-Pro Plus version 6.0 (Media Cybernetics, Inc., Rockville, MD, USA).

Cell cycle analysis. After 4 days of lentivirus transfection, Y79 and SO-RB50 cells (1x10 $10^{6}$ cells per well) were harvested and washed twice with cold PBS, followed by fixation with ice-cold $70 \%$ ethanol overnight at $4^{\circ} \mathrm{C}$. After washing twice with PBS containing 0.5\% Triton X-100, the cells were incubated with $50 \mu \mathrm{g} / \mathrm{ml}$ propidium iodide (PI) and $50 \mu \mathrm{g} / \mathrm{ml}$ RNaseA (both BD Biosciences, San Jose, CA, USA) for $30 \mathrm{~min}$ at room temperature. The cells were then analyzed using a fluorescence-activated cell sorting (FACS) system with a FACSCalibur with CellQuest software (version 5.1) (both BD Biosciences). The data were analyzed using the ModFit software (version 4.0; Verity Software House, Topsham, ME, USA).

Statistical analysis. All observations were confirmed by at least three independent experiments. Quantitative data are expressed as the mean \pm standard deviation. Two-tailed Student's t-test was performed for paired samples. $\mathrm{P}<0.05$ was considered to indicate a statistically significant difference.

\section{Results}

Nek7 is abnormally upregulated in human retinoblastoma cell lines. To investigate the biological function of Nek7 in human retinoblastoma, the expression of Nek7 was examined in several human retinoblastoma cell lines and normal RPE cells. The results identified that the mRNA expression level of Nek7 was commonly upregulated in human retinoblastoma cell lines (Y79, SO-RB50 and WERI-RB1), as compared with that in RPE cells (Fig. 1A; P<0.001). To further confirm this finding, the protein level of Nek7 was determined by western blot analysis. Consistently, the Nek7 protein level was higher in retinoblastoma cell lines compared with that in normal RPE cells (Fig. 1B). Thus, these results suggested that Nek7 may be involved in retinoblastoma development. Notably, Y79 and
A

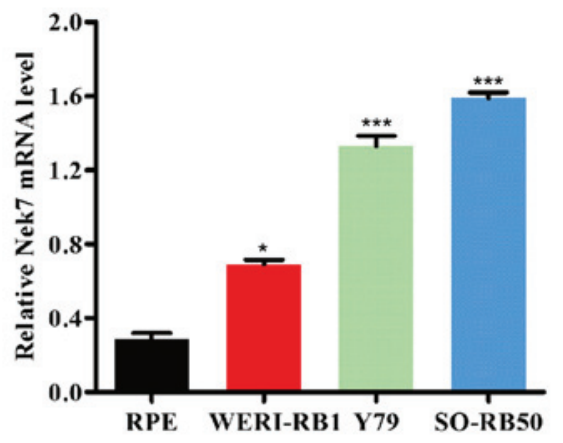

B

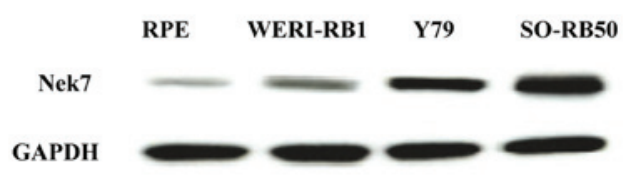

Figure 1. Nek7 was abnormally upregulated in human retinoblastoma cell lines. (A) RT-qPCR and (B) western blot assays were used to determine the mRNA and protein expression levels of Nek7, respectively. Compared with normal RPE cells, high expression of Nek7 was observed in retinoblastoma Y79, SO-RB50 and WERI-RB1 cells. Nek7 mRNA and protein levels in SO-RB50 and Y79 were relatively higher compared with WERI-RB1 cells. $\beta$-actin and GAPDH were used as the internal controls in RT-qPCR and western blot assays, respectively. Experiments were repeated three times. ${ }^{*} \mathrm{P}<0.05$ and $^{* * *} \mathrm{P}<0.001$, vs. normal RPE cells. Nek7, NIMA-related kinase-7; RT-qPCR, reverse transcription-quantitative polymerase chain reaction; RPE, retinal pigment epithelium.

SO-RB50 cells that demonstrated higher Nek7 expression were selected for subsequent analysis.

Lentivirus-mediated shRNA transfection strongly suppresses Nek7 expression in Y79 and SO-RB50 cells. The Y79 and SO-RB50 cells were transfected with shNC, shNek7-1 or shNek-2 lentivirus particles. As shown in Fig. 2A, the majority of cells presented GFP-positive signals following shRNA transfection (with shNC, shNek7-1 and shNek7-2), indicating satisfying transfection efficiency. In order to verify that the Nek7 gene was silenced by the lentivirus, the mRNA and protein levels in Y79 and SO-RB50 cells were assessed using RT-qPCR and western blot assays, respectively. In Y79 cells, the mRNA and protein levels of Nek7 were significantly decreased in shNek7-1 and shNek-2 groups compared with the shNC group (Fig. 2B and C). Similarly, the expression of Nek7 was strongly reduced in shNek7-1 and shNek-2 groups compared with the shNC group in SO-RB50 cells (Fig. 2D and E). These results suggest that lentivirus-mediated Nek7 shRNA significantly downregulated Nek7 expression in Y79 and SO-RB50 cells. Notably, the knockdown efficacy of the shNek7-1 vector was more evident in comparison with that of shNek-2 in the Y79 and SO-RB50 cells.

Knockdown of Nek7 inhibits proliferation and colony formation of Y79 and SO-RB5O cells. To investigate the effect of Nek7 knockdown on cell growth, Y79 and SO-RB50 cells transfected with shNC, shNek7-1 and shNek-2 were subjected to MTT assay The results revealed that the viability of Y79 cells was markedly decreased, 
A
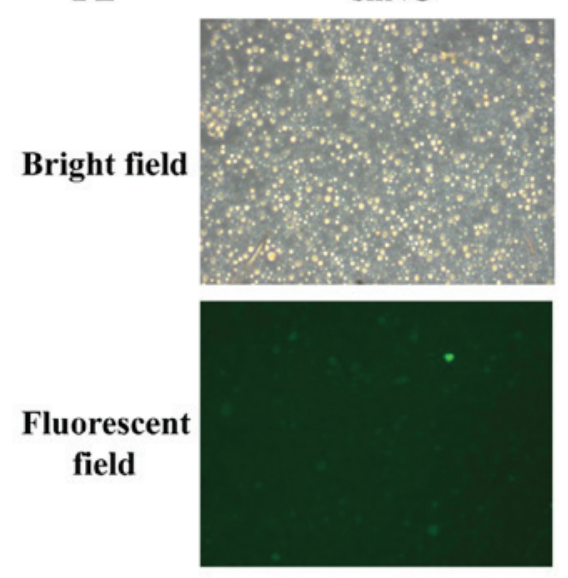

B

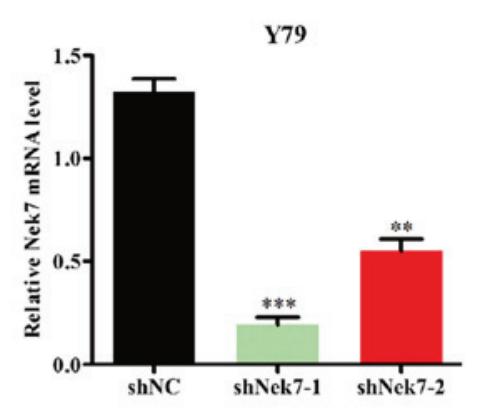

D

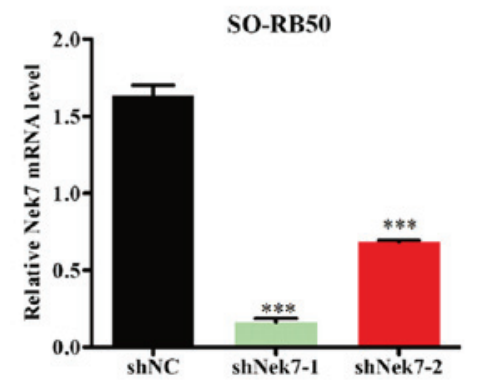

shNek7-1
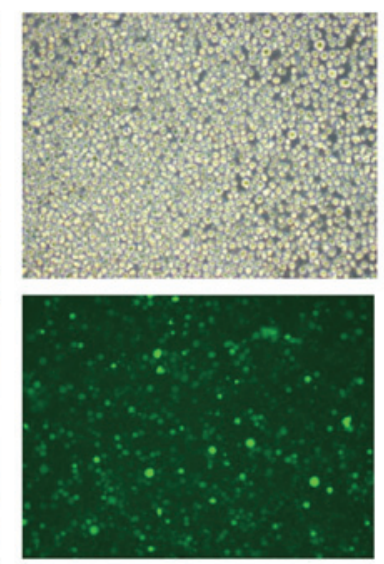

shNek7-2
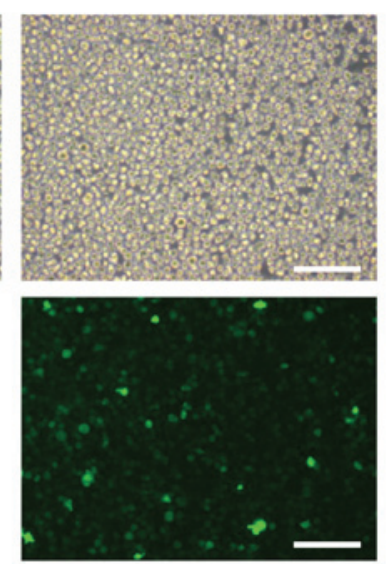

C

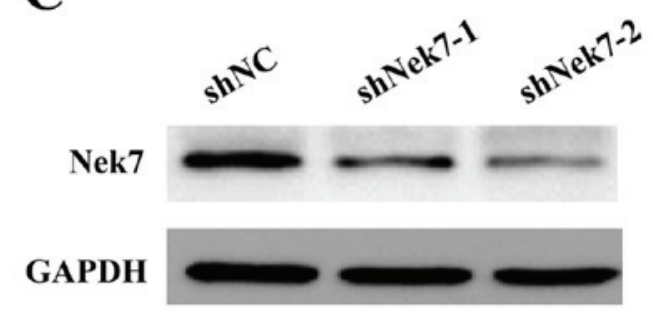

$\mathbf{E}$

Nek7

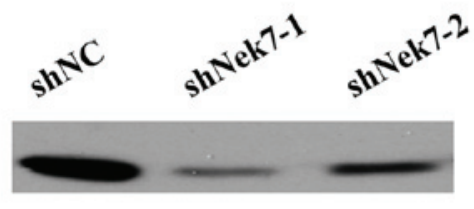

GAPDH

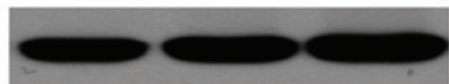

Figure 2. Knockdown of Nek7 in Y79 and SO-RB50 cells by lentivirus-delivered shNek7-1 and shNek7-2. (A) Detection of lentiviral transfection efficiency. Y79 and SO-RB50 cells were transfected with shNC, shNek7-1 or shNek-2, and bright field (top) or green fluorescence protein-staining (bottom) images were obtained at $96 \mathrm{~h}$ after transfection. Scale bar, $100 \mu \mathrm{m}$ and magnification, x200. (B) Nek7 mRNA expression and (C) Nek7 protein expression determined by RT-qPCR and western blot analysis, respectively, in Y79 cells transfected with shNC, shNek7-1 and shNek7-2. (D) Nek7 mRNA expression and (E) Nek7 protein expression determined by RT-qPCR and western blot analysis, respectively, in SO-RB50 cells transfected with shNC, shNek7-1 and shNek7-2. Experiments were repeated three times. ${ }^{* *} \mathrm{P}<0.01$ and ${ }^{* * * *} \mathrm{P}<0.001$ vs. shNC group. Nek7, NIMA-related kinase-7; RT-qPCR, reverse transcription-quantitative polymerase chain reaction; shNC, negative control short hairpin RNA.

with the proliferation ability decreasing by $73.3 \%$ in the shNek7-1 group and 40.2\% in the shNek7-2 group $(\mathrm{P}<0.001$, Fig. 3A) at 5 days after transfection. Furthermore, it was observed that the proliferation ability reduced by $75.9 \%$ in shNek7-1-transfected and 52.6\% in shNek7-2-transfected SO-RB50 cells $(\mathrm{P}<0.001$; Fig. $3 \mathrm{~B})$ at 5 days. Given the aforementioned findings, it is suggested that shNek7-1 had a more powerful effect on inhibiting the cell proliferation ability as compared with shNek7-2.

The colony formation ability of Y79 and SO-RB50 cells was also determined in order to gain additional insight into the effect of shNek7-1 on cell proliferation. For Y79 cells, shNek7-1 resulted in smaller colony numbers compared with the shNC group (Fig. 3C and D; $\mathrm{P}<0.01$ ). Similarly, smaller colony numbers were identified in SO-RB50 cells transfected with shNek7-1 (Fig. 3E and F; P<0.001). These results indicated that silencing Nek7 may inhibit the cell proliferation and colony formation abilities of Y79 and SO-RB50 cells, which correlates with the formation of human retinoblastoma.

Knockdown of Nek7 arrests the cell cycle progression of $Y 79$ and SO-RB50 cells. To examine whether the growth suppression effect of Nek7 knockdown was associated with cell cycle regulation, flow cytometry with PI staining for cell cycle distribution profiles was performed in Y79 and SO-RB50 cells transfected with shNek7-1 (Fig. 4A). As demonstrated in the FACS analysis in Fig. 4B, the proportion of cells in the $S$ phase was markedly decreased (from 27.16 to $18.24 \%$; $\mathrm{P}<0.001$ ), whereas the proportion of cells was increased in the G0/G1 (from 61.40 to $69.72 \%$; $\mathrm{P}<0.001$ ) and 
A

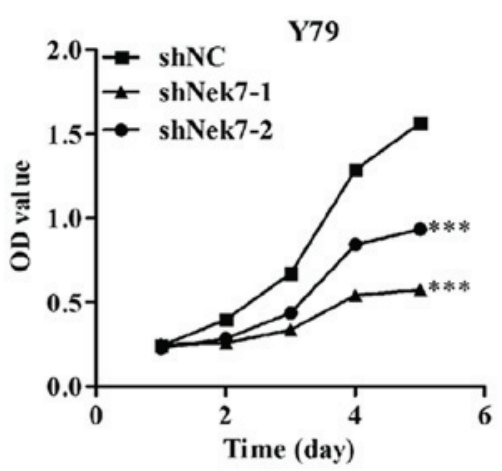

C

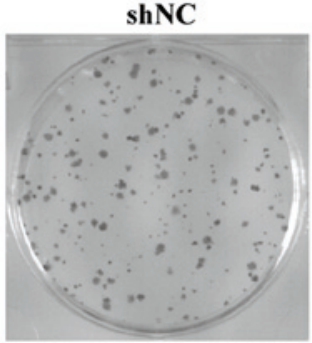

$\mathbf{E}$

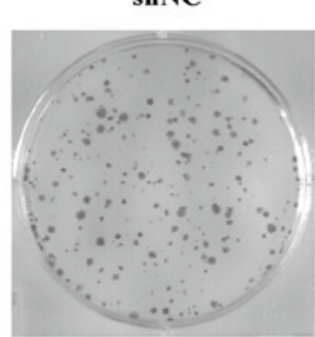

shNek7-1

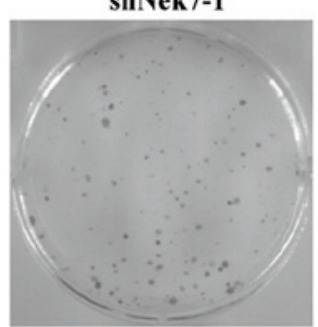

shNek7-1

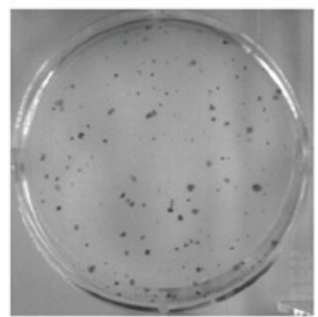

B

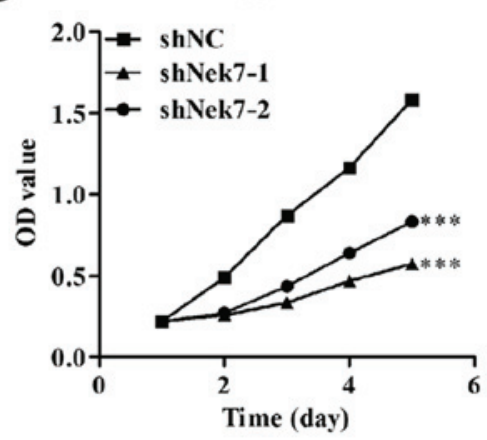

D

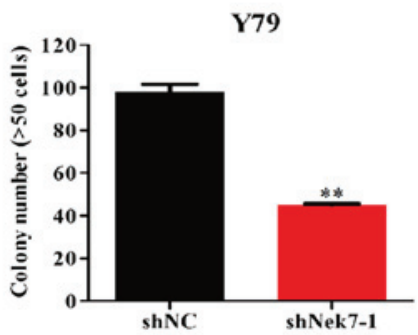

F

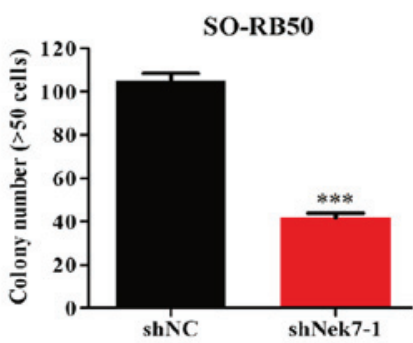

Figure 3. Knockdown of Nek7 inhibited the growth ability and proliferation of Y79 and SO-RB50 cells in vitro. Cell growth was determined using MTT assay. Growth curves of (A) Y79 cells and (B) SO-RB50 cells transfected with shNC, shNek7-1 and shNek7-2. Cells transfected with shNek7-1 and shNek-2 presented reduced growth compared with the shNC group. Cell proliferation was detected using colony formation assay. (C) Images representing the size of colonies and (D) the quantified number of colonies in Y79 cells transfected with shNek7-1and shNC. (E) Images representing the size of colonies and (F) the quantified number of colonies in SO-RB50 cells transfected with shNek7-1and shNC. The number of colonies was significantly decreased in Y79 and SO-RB50 cells transfected with shNek7-1 compared with the shNC-transfected cells. Experiments were repeated three times ${ }^{* *} \mathrm{P}<0.01$ and ${ }^{* * *} \mathrm{P}<0.001$ vs. shNC group. Nek7, NIMA-related kinase-7; shNC, negative control short hairpin RNA.

G2/M (from 11.43 to $12.04 \%$ ) phases in Y79 cells transfected with shNek7-1 compared with the shNC group. A similar phenomenon was also observed in SO-RB50 cells transfected with shNek7-1, in which the proportion of cells in the $\mathrm{S}$ phase was significantly reduced (from 17.58 to $13.11 \%$; $\mathrm{P}<0.01$ ), while the proportion of cells was markedly enhanced in the G0/G1 (from 61.79 to 64.03\%; P<0.01) and $\mathrm{G} 2 / \mathrm{M}$ (from 20.63 to $22.87 \%$; $\mathrm{P}<0.001$ ) phases, compared with the shNC group (Fig. 4). These results suggest that the growth suppression mediated by shNek7-1 may be, in part, through arrest of the cell cycle in $\mathrm{G} 0 / \mathrm{G} 1$ phase.

Knockdown of Nek7 modifies the expression of cell cycleassociated proteins. In order to further clarify the mechanism underlying the antiproliferative effect of Nek7 knockdown, several downstream cell cycle-associated regulators in Y79 and SO-RB50 cells were analyzed by western blot assay. The data revealed that the cell cycle checkpoint kinase CDK2 was clearly decreased in Y79 cells transfected with shNek7-1. In addition, cyclin D1 and cyclin E, which function as regulators of CDKs, were evidently decreased by silenced Nek7 (Fig. 5A). Similar results were also observed for SO-RB50 cells (Fig. 5B). These results clearly indicated that Nek7 silencing may inhibit the proliferation of Y79 and SO-RB50 cells by downregulating the expression of cell cycle-associated proteins.

\section{Discussion}

Nek7, a member of the Nek protein kinase family, is involved in cell cycle regulation (21). Recently, the oncogenic potential of Nek7 has been recognized in various types of cancer, including gallbladder cancer (16) and hepatocellular carcinoma (17), suggesting that Nek7 may serve a crucial role in retinoblastoma development. Based on this speculation, the present study examined the Nek7 expression in retinoblastoma cell lines. Compared with the normal RPE cells, Nek7 was highly expressed in retinoblastoma cells, suggesting that the expression pattern of Nek7 correlated with retinoblastoma progression. In agreement with the present study findings, a 

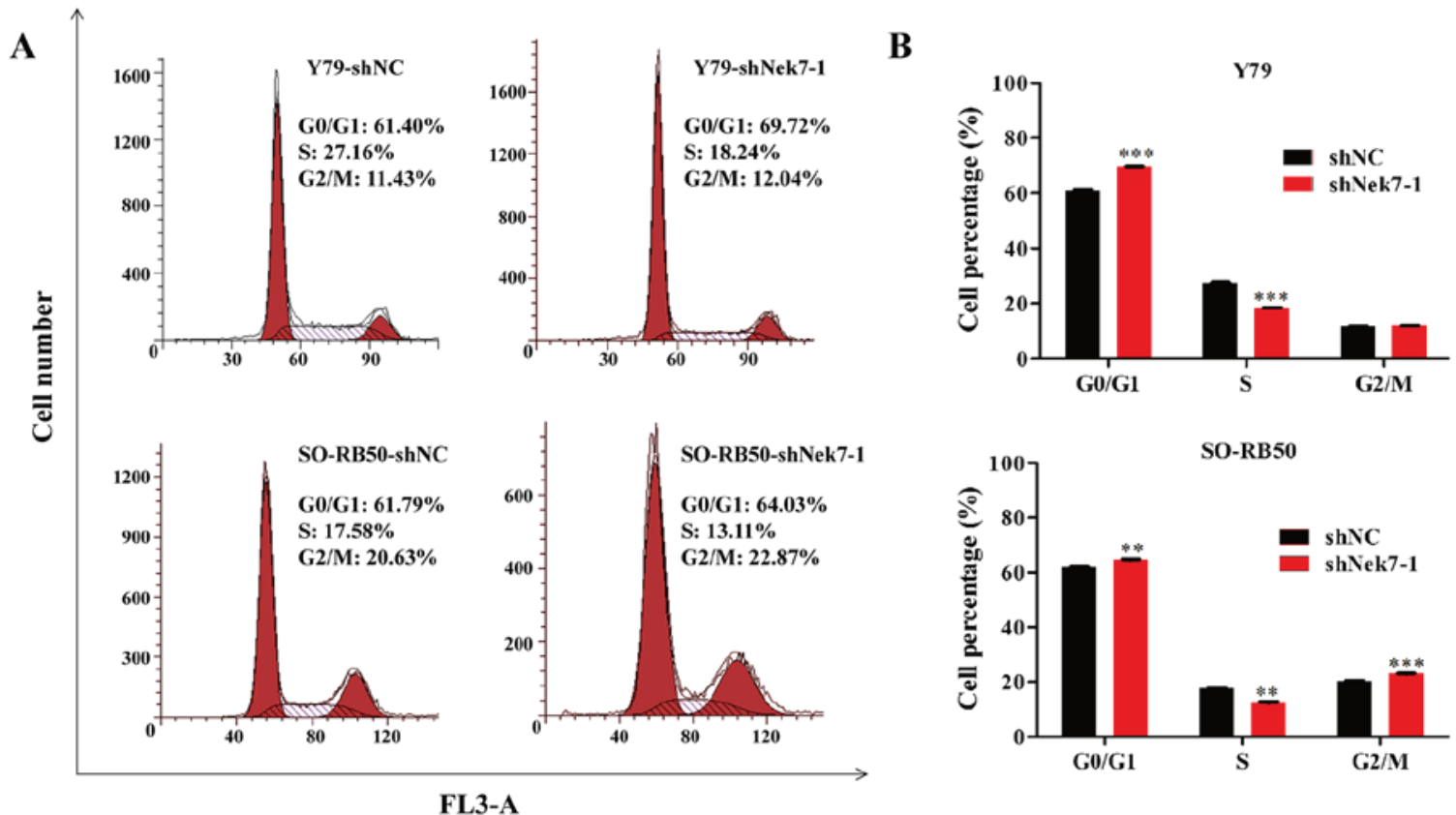

Figure 4. Knockdown of Nek7 blocks the cell cycle progression of Y79 and SO-RB50 cells. Cell cycle distribution was analyzed by flow cytometry. (A) Representative images of shNC-transfected and shNek7-1-transfected Y79 and SO-RB50 cells are shown, as determined by fluorescence-activated cell sorting analysis. (B) Proportion of cells in the different cell cycle phases in Y79 and SO-RB50 cells transfected with shNC and shNek7-1. Experiments were repeated three times. ${ }^{* *} \mathrm{P}<0.01$ and ${ }^{* * * *} \mathrm{P}<0.001$ vs. shNC group. Nek7, NIMA-related kinase-7; shNC, negative control short hairpin RNA.

A

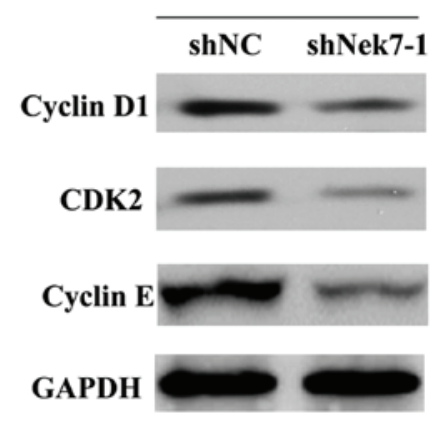

B

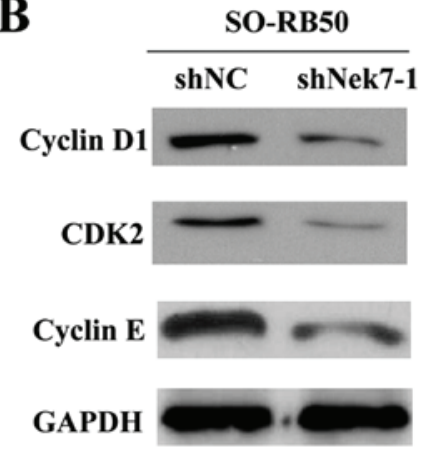

Figure 5. Knockdown of Nek7 effects on cell cycle regulatory protein expression, determined by western blot analysis. The levels of CDK2, cyclin D1 and cyclin E protein in (A) Y79 cells and (B) SO-RB50 cells transfected with shNC and shNek7-1 are shown. GAPDH was used as an internal loading control. Experiments were repeated three times. Nek7, NIMA-related kinase-7; shNC, negative control short hairpin RNA; CDK2, cyclin-dependent kinase 2.

meta-analysis by Kooi et al (18) previously indicated that Nek7 presented the highest mean expression among the identified candidate genes in retinoblastoma.

The current study further explored the biological role of Nek7 in retinoblastoma cells and identified that Nek7 silencing significantly inhibited the growth and colony formation ability of retinoblastoma cells. Accumulating evidence has demonstrated that Nek7 is located at the centrosome and cytoplasm $(21,22)$, and has great influence during the steps of mitosis and cytokinesis (23). In addition, Nek7 serves an essential role in centriole duplication in the cell mitosis phase (24). Therefore, the present study speculated that knockdown of Nek7 may suppress retinoblastoma cell proliferation through the deregulation of the cell cycle. To confirm this hypothesis, the effects of Nek7 silencing on retinoblastoma cell cycle progression were analyzed using flow cytometry analysis. As expected, knockdown of Nek7 induced cell cycle arrest at G0/G1 phase in two retinoblastoma cell lines, Y79 and SO-RB50.

Mechanistic analysis in the present study demonstrated that downregulation of Nek7 resulted in decreased CDK2, cyclin D1 and cyclin E expression levels in retinoblastoma cells. The G1/S transition is considered as one of the main checkpoints of cell cycle progression by regulating the initiation and completion of DNA replication, which is controlled by the activation of CDK/cyclin complexes $(25,26)$. Since the combined activity of the CDK2/cyclin E complex serves an important role in initiating the progression from G1 phase to S phase $(27,28)$, it is possible that the knockdown of Nek7 expression suppresses retinoblastoma cell proliferation by downregulating CDK2, cyclin E and cyclin D1 expression.

In conclusion, the results of the present study demonstrated that knockdown of Nek7 suppresses retinoblastoma cell proliferation by induction of cell cycle arrest at G0/G1 phase. In 
addition, Nek7 may be involved in retinoblastoma progression by regulating the expression of $\mathrm{CDK} /$ cyclin complexes. These findings provide an experimental basis for the knockdown of Nek7 as a novel molecular therapeutic target for malignant retinoblastoma.

\section{References}

1. Houston SK, Murray TG, Wolfe SQ and Fernandes CE: Current update on retinoblastoma. Int Ophthalmol Clin 51: 77-91, 2011.

2. Mendoza PR and Grossniklaus HE: Therapeutic options for retinoblastoma. Cancer Control 23: 99-109, 2016.

3. Knudson AG Jr: Mutation and cancer: Statistical study of retinoblastoma. Proc Natl Acad Sci USA 68: 820-823, 1971.

4. Saiju R and Duwal S: Bilateral retinoblastoma in early infancy. Nepal J Ophthalmol 5: 124-128, 2013.

5. Friedman DL, Himelstein B, Shields CL, Shields JA, Needle M, Miller D, Bunin GR and Meadows AT: Chemoreduction and local ophthalmic therapy for intraocular retinoblastoma. J Clin Oncol 18: 12-17, 2000.

6. Benz MS, Scott IU, Murray TG, Kramer D and Toledano S: Complications of systemic chemotherapy as treatment of retinoblastoma. Arch Ophthalmol 118: 577-578, 2000.

7. O'Connell MJ, Krien MJ and Hunter T: Never say never. The NIMA-related protein kinases in mitotic control. Trends Cell Biol 13: 221-228, 2003.

8. Manning G, Whyte DB, Martinez R, Hunter T and Sudarsanam S: The protein kinase complement of the human genome. Science 298: 1912-1934, 2002

9. Fry AM and Nigg EA: Cell cycle. The NIMA kinase joins forces with Cdc2. Curr Biol 5: 1122-1125, 1995.

10. Osmani AH, Mcguire SL and Osmani SA: Parallel activation of the NIMA and p34cdc2 cell cycle-regulated protein kinases is required to initiate mitosis in A. nidulans. Cell 67: 283-291, 1991.

11. Lu KP and Hunter T: Evidence for a NIMA-like mitotic pathway in vertebrate cells. Cell 81: 413-424, 1995.

12. Kandli M, Feige E, Chen A, Kilfin G and Motro B: Isolation and characterization of two evolutionarily conserved murine kinases (Nek6 and Nek7) Related to the fungal mitotic regulator, NIMA. Genomics 68: 187-196, 2000.

13. Feige E and Motro B: The related murine kinases, Nek6 and Nek7, display distinct patterns of expression. Mech Dev 110: 219-223, 2002

14. Yin MJ, Shao L, Voehringer D, Smeal T and Jallal B: The serine/threonine kinase Nek6 is required for cell cycle progression through mitosis. J Biol Chem 278: 52454-52460, 2003.

15. Yissachar N, Salem H, Tennenbaum T and Motro B: Nek7 kinase is enriched at the centrosome, and is required for proper spindle assembly and mitotic progression. FEBS Lett 580: 6489-6495, 2006.
16. Wang R, Song Y, Xu X, Wu Q and Liu C: The expression of Nek7, FoxM1, and Plk1 in gallbladder cancer and their relationships to clinicopathologic features and survival. Clin Transl Oncol 15: 626-632, 2013

17. Lei Z, Wang Z, Xu X, Wan Y, Qu K, Fan H, Chen Q, Sun X and Liu C: Nek7 is overexpressed in hepatocellular carcinoma and promotes hepatocellular carcinoma cell proliferation in vitro and in vivo. Oncotarget 7: 18620-18630, 2016.

18. Kooi IE, Mol BM, Massink MP, de Jong MC, de Graaf P, van der Valk P, Meijers-Heijboer H, Kaspers GJ, Moll AC, Te Riele H, et al: A meta-analysis of retinoblastoma copy numbers refines the list of possible driver genes involved in tumor progression. PLoS One 11: e0153323, 2016.

19. Livak KJ and Schmittgen TD: Analysis of relative gene expression data using real-time quantitative PCR and the 2(-Delta Delta C(T)) method. Methods 25: 402-408, 2001.

20. Scherr M, Chaturvedi A, Battmer K, Dallmann I, Schultheis B, Ganser A and Eder M: Enhanced sensitivity to inhibition of SHP2, STAT5, and Gab2 expression in chronic myeloid leukemia (CML). Blood 107: 3279-3287, 2006.

21. O'Regan L and Fry AM: The Nek6 and Nek7 protein kinases are required for robust mitotic spindle formation and cytokinesis. Mol Cell Biol 29: 3975-3990, 2009.

22. Kim S, Lee K and Rhee K: NEK7 is a centrosomal kinase critical for microtubule nucleation. Biochem Biophys Res Commun 360: 56-62, 2007.

23. Salem H, Rachmin I, Yissachar N, Cohen S, Amiel A, Haffner R, Lavi L and Motro B: Nek7 kinase targeting leads to early mortality, cytokinesis disturbance and polyploidy. Oncogene 29: 4046-4057, 2010.

24. Kim S, Kim S, Kim S and Rhee K: NEK7 is essential for centriole duplication and centrosomal accumulation of pericentriolar material proteins in interphase cells. J Cell Sci 124: 3760-3770, 2011.

25. Massagué J: G1 cell-cycle control and cancer. Nature 432: 298-306, 2004.

26. Malumbres M and Barbacid M: Cell cycle, CDKs and cancer: $A$ changing paradigm. Nat Rev Cancer 9: 153-166, 2009.

27. Cicenas J, Kalyan K, Sorokinas A, Jatulyte A, Valiunas D, Kaupinis $A$ and Valius M: Highlights of the latest advances in research on CDK inhibitors. Cancers (Basel) 6: 2224-2242,2014.

28. Wei L, Lin J, Wu G, Xu W, Li H, Hong Z and Peng J: Scutellaria barbata D. Don induces G1/S arrest via modulation of p53 and Akt pathways in human colon carcinoma cells. Oncol Rep 29: 1623-1628, 2013

This work is licensed under a Creative Commons Attribution-NonCommercial-NoDerivatives 4.0 International (CC BY-NC-ND 4.0) License. 